\title{
RecQ helicases queuing with Srs2 to disrupt Rad51 filaments and suppress recombination
}

\author{
Dana Branzei $^{2}$ and Marco Foiani ${ }^{1}$ \\ Fondazione Italiana Ricerca sul Cancro (FIRC) Institute of Molecular Oncology Foundation, 20139 Milan, Italy; \\ Dipartimento di Scienze Biomolecolari e Biotecnologie, Università degli Studi di Milano, 20133 Milan, Italy
}

Homologous recombination (HR) is an important mechanism for the maintenance of genome integrity. HR functions to repair double-strand breaks (DSBs) and single-strand gaps formed during replication or created by DNA damaging agents or from processing DNA lesions. In addition, HR is implicated in the restart of damaged replication forks and functions in telomere length maintenance in cells lacking telomerase. Increasing evidence suggests that HR plays an important role in cancer prevention (Thompson and Schild 2002; Sung and Klein 2006). However, recombination can also be harmful and have oncogenic and mutagenic consequences. It is known that inappropriate or untimely recombination can generate damaging genome rearrangements, such as somatic loss of heterozygosity (LOH), chromosome deletions, inversions, or translocations. Therefore, cells have evolved specific mechanisms to control recombination and to coordinate HR with other responses to DNA damage as well as with replication and progression through the cell cycle. Indeed, several cancer-prone genetic diseases, including the ones caused by mutations in human RecQ helicases, are associated with HR dysfunction. In this issue of Genes \& Development, two studies by Bugreev et al. (2007b) and $\mathrm{Hu}$ et al. (2007) demonstrate a novel function for two human RecQ helicases, BLM and RECQL5, in regulating an early step of $\mathrm{HR}$, which is related to their role in protecting genome stability.

An important early step of all $\mathrm{HR}$ reactions is to use a Rad51 presynaptic filament on single-stranded DNA (ssDNA) (Fig. 1A) to invade a homologous duplex, giving rise to a three-stranded structure called a D-loop (Fig. 1B). HR can then bifurcate into two main subpathways called DSB repair (DSBR) and synthesis-dependent strand annealing (SDSA) (Fig. 2; Allers and Lichten 2001; Hunter and Kleckner 2001). In DSBR, the second DSB end can be captured to form an intermediate with two Holliday junctions (HJs), usually called a double $\mathrm{HJ}$ (dHJ),

Correspondence.

${ }^{1}$ E-MAIL marco.foiani@ifom-ieo-campus.it; FAX 39-02-574303231.

${ }^{2}$ E-MAIL dana.branzei@ifom-eo-campus.it; FAX 39-02-574303231.

Article is online at http://www.genesdev.org/cgi/doi/10.1101/gad.1624707. that can be resolved at the HJs by endonucleases to crossover and noncrossover products (Paques and Haber 1999; Sung and Klein 2006), or dissolved to noncrossover products by the concerted actions of a helicase and topoisomerase (Fig. 2; see below; Sung and Klein 2006 and references therein). In SDSA, however, the extended D-loop is dissolved, likely by a DNA helicase, and the extended single-stranded end anneals to the ssDNA of the other break end, followed by gap-filling DNA synthesis and ligation, to restore a contiguous chromosome always in noncrossover configuration (Fig. 2; Paques and Haber 1999; Sung and Klein 2006). Crossover is essential for proper chromosome disjunction during meiosis, but it is likely suppressed in mitotic cells as it can lead to LOH and chromosome rearrangements. Conceivably, cells are endowed with systems that regulate HR so as to generate exclusively or predominantly noncrossover products. Results of several studies have suggested that an important enzymatic function in both promoting SDSA and regulating $\mathrm{dHJ}$ resolution to noncrossover products is achieved through the BLM helicase (Fig. 2; see below; Adams et al. 2003; Wu and Hickson 2003; McVey et al. 2004; Johnson-Schlitz and Engels 2006).

BLM is a member of the highly conserved RecQ family of helicases with crucial roles in the maintenance of genome stability. Consequently, no eukaryotic organism has been found that lacks a RECQ gene. While Saccharomyces cerevisiae has one RecQ gene (Sgs1), humans have five (WRN, BLM, RECLQ4, RECQL1, and RECQL5). Deficiencies in three of them-WRN, BLM, and RECQL4cause WS (Werner syndrome), BS (Bloom syndrome), and RTS (Rothmund-Thomson syndrome), respectively. These genetic disorders are characterized by cancer predisposition, premature aging, and/or developmental abnormalities (Sharma et al. 2006; Hanada and Hickson 2007).

\section{BLM's role in controlling HR and suppressing SCE formation}

One of the most characteristic symptoms of BS patients is a dramatic cancer predisposition. BS cells have an abnormally high rate of $\mathrm{HR}$, and about a 10-fold increased 
A

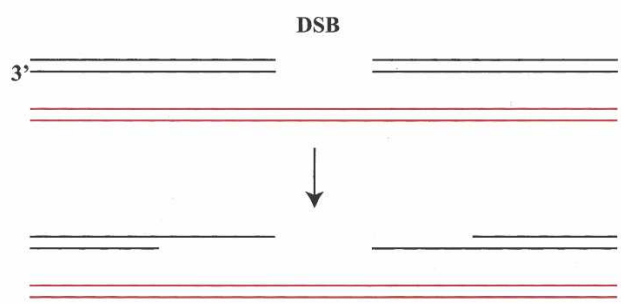

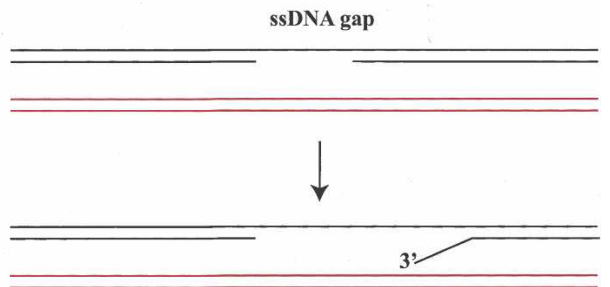

B
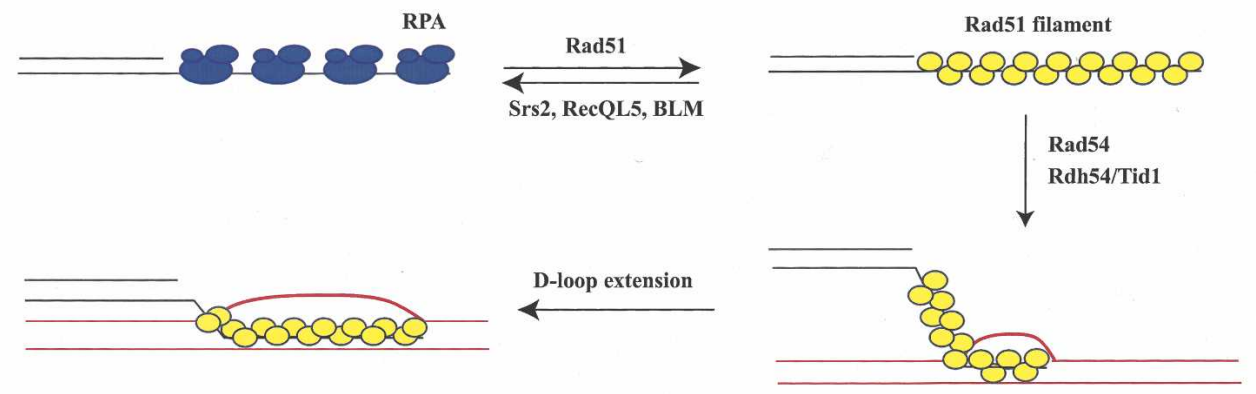

D-loop formation

Figure 1. Schematic representation of 3 '-overhang formation and strand invasion. $(A)$ DSBs or DNA gaps are processed nucleolytically or by the action of DNA helicases to expose $3^{\prime}$-overhangs of ssDNA. $(B)$ The ssDNA of 3 '-overhangs or generated at stalled forks is immediately coated by RPA, but RPA can be removed and replaced by Rad51 with the help of mediator proteins such as Rad52, Rad55, and Rad57 to form what is a called the Rad51 presynaptic filament. This process is counteracted by helicases such as Srs2, BLM, and RECQL5 that disrupt the Rad51 filaments. Once formed, the Rad51 filament can engage in a homology search and invade the homologous region in the duplex to form a DNA joint called a D-loop. The D-loop can be then extended through DNA synthesis.

rate of sister chromatid exchanges (SCEs), a feature that is used as a molecular diagnosis of the disorder (Ray and German 1984; Hanada and Hickson 2007). When HR uses the homologous chromosome instead of the sister chromatid as a repair template, LOH may occur. Indeed, mutations in both human $B L M$ and mouse $B l m$ also result in elevated rates of LOH (German 1993; Luo et al. 2000; Goss et al. 2002). Furthermore, SCEs using nonidentical repeat sequences or occurring unequally between identical sequences can lead to chromosome duplications and deletions, an important source of genomic instability. Although the mechanism of SCE formation is not fully understood, previous studies suggest that a large part of SCEs require HR for their formation (Sonoda et al. 1999; Wang et al. 2000). Hence, it follows that one important function of BLM is to suppress HR events leading to SCEs and LOH. Indeed, the relevance of BLM suppressing SCEs in cancer avoidance is well established (German 1993; Luo et al. 2000; Goss et al. 2002). Since SCEs represent crossover events, conceivably BLM may act to suppress their formation through D-loop unwinding of unwanted strand invasion events (Bachrati et al. 2006), by promoting SDSA (Adams et al. 2003), or in DSBR by favoring the formation of noncrossover products (Fig. 2; Wu and Hickson 2003). Studies in Drosophila have suggested that BLM is required for SDSA (Adams et al. 2003), perhaps by acting downstream from strand invasion to unwind the D-loop intermediate and free the newly synthesized strand (McVey et al. 2004).
This model explains that in the absence of DmBlm, alternative pathways of D-loop disassembly result in short repair synthesis tracts or flanking deletions.

In this issue, Bugreev et al. (2007b) show biochemical evidence using in vitro reconstitution of $\mathrm{HR}$ reactions that BLM might indeed promote late steps of SDSA through D-loop disruption and by stimulating DNA repair synthesis of DNA polymerase $\eta$ (Fig. 2). However, there is also recent evidence that argues against the role of BLM in SDSA based on observations that BLM deficiency leads to structural alterations in both the template and the donor sequences, which would not be predicted by the SDSA model (Johnson-Schlitz and Engels 2006). Rather, analysis of the template disruptions that occur in the absence of BLM points to a failure to dissolve dHJs (Johnson-Schlitz and Engels 2006), consistent with a model previously proposed to explain the role of BLM in promoting formation of noncrossover recombinants (Wu and Hickson 2003). In this $\mathrm{dHJ}$ dissolution model (Fig. 2), it is proposed that BLM drives the convergence of the $\mathrm{dHJ}$ s via branch migration into a hemicatenane structure, which is resolved using the ssDNA passage activity of Top3 $\alpha$ (Wu and Hickson 2003). Also, consistent with this biochemical activity of Blm, genetic studies in yeast have brought support to this model (Ira et al. 2003; Robert et al. 2006) and shown that mutations in Sgs 1 and Top3 lead to accumulation of X-shaped DNA structures at damaged replication forks that might represent hemicatenane-like molecules (Liberi et al. 2005). 


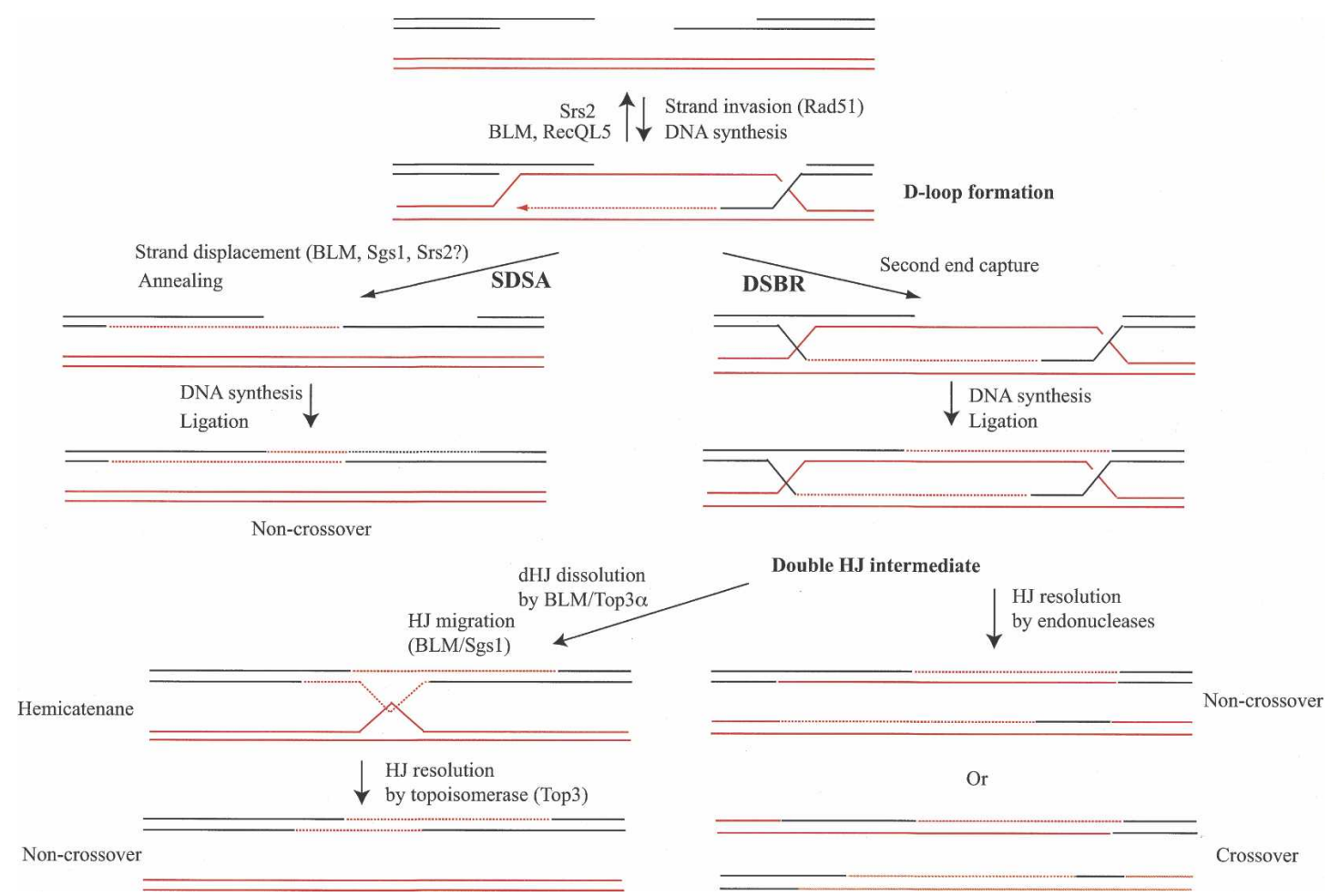

Figure 2. DSBR by SDSA and DSBR pathways. Resected DSBs expose 3' ssDNA overhangs that initiate strand invasion followed by DNA synthesis. Strand displacement of this intermediate by a DNA helicase (BLM, Sgs1, and possibly Srs2) channels the reaction toward SDSA. However, if the second DSB end is captured, an intermediate with two HJs, called a dHJ, forms. A dHJ can be dissolved by means of a helicase (BLM) and topoisomerase (Top3 $\alpha$ ) to give a noncrossover product, or resolved at HJs by endonucleases to give both crossover and noncrossover recombinants.

\section{RecQ helicases and Srs2 in suppressing recombination}

Mutations in human RECQL1 and RECQL5 are not yet linked to a disease, but studies using DT40 cells or knockout mice suggested that deficiencies in these genes might also predispose individuals to cancer or chromosome instability disorders (Wang et al. 2003; Sharma et al. 2006; Hanada and Hickson 2007). In this issue, Hu et al. (2007) provide evidence that deletion of Recql5 in mice results in increased susceptibility to cancer and gross chromosomal rearrangement (GCR) accumulation, likely due to a failure to regulate $\mathrm{HR}$, and show through biochemical experiments that RecQL5 functions to disrupt Rad51 filaments thereby attenuating HR initiation. RecQL5 is not the only RecQ helicase capable of such a feat. In fact, Bugreev et al. (2007b) demonstrate that in addition to its previously discovered anti-recombination activities (see Sharma et al. 2006; Hanada and Hickson 2007), BLM can also function to disrupt Rad51 filaments, thereby suppressing $\mathrm{HR}$ at an early stage.

A potent and well-studied mechanism to control recombination in yeast involves the Srs2 protein (Sung and Klein 2006). Srs2 is a helicase with similarities to the bacterial UvrD/Rep helicases (Rong and Klein 1993). Mutations in the SRS2 gene lead to increased recombination and suppression of the damage sensitivity of post-replication repair mutants in a manner dependent on HR genes (Aboussekhra et al. 1989; Schiestl et al.
1990). These genetic observations suggested that Srs2 functions to restrict recombination, and, indeed, later biochemical evidence provided the mechanistic basis of the Srs2 function, by revealing its ability to bind Rad51 and disrupt Rad51 presynaptic filaments (Krejci et al. 2003; Veaute et al. 2003). So far, an Srs2 ortholog has not yet been found in other eukaryotes, although the newly identified Fbh1 helicase shows some structure similarities to Srs2 in the helicase domain (Morishita et al. 2005; Osman et al. 2005; Chiolo et al. 2007). Genetic studies conducted in Schizosaccharomyces pombe and chicken DT40 cells suggest that Fbh1 plays a role in processing HR intermediates and in suppressing SCE formation (Morishita et al. 2005; Osman et al. 2005; Kohzaki et al. 2007). However, it remained unclear whether disruption of the Rad51 presynaptic filament represents a significant mechanism of HR attenuation and control in higher eukaryotes.

The findings reported in this issue by $\mathrm{Hu}$ et al. (2007) and Bugreev et al. (2007b) are particularly important as they show that two human RecQ helicases, BLM and RecQL5, can also act as translocases to dislodge Rad51 from presynaptic filaments, thus functioning similarly to Srs2 to suppress the initiation of HR. In fact, previous genetic data suggested a possible functional overlap between Srs2 and the yeast RecQ helicase Sgs1 in counteracting recombination. For instance, in sgs1 srs2 double mutants, Rad51-mediated recombination causes cell 
death (Gangloff et al. 2000), and Sgs1 overexpression can suppress recombination and repair defects of srs2 (Mankouri et al. 2002; Ira et al. 2003). These data suggest that in srs2 cells, potentially toxic recombination intermediates may form, which are substrates for Sgs1. Srs2 becomes essential also in other mutant contexts such as the one of rad54, encoding a double-stranded DNA (dsDNA) translocase that both promotes D-loop formation and enhances DNA branch migration (Sung and Klein 2006). In addition, as in the case of sgs 1 srs2, the synthetic lethality of srs2 rad54 is suppressed by rad51 mutation (Rong and Klein 1993; Sung and Robberson 1995). These observations led to the current view that holds that Srs2 disrupts Rad51 nucleofilaments essentially when subsequent recombination steps are impaired. Sgs1 and Srs2 do not seem to be redundant, and while they could sometimes substitute for each other, it is conceivable that they may act preferentially at different steps in HR or, for instance, at different stages in the cell cycle or with respect to the timing of DNA replication. While BLM likely represents the Sgs1 ortholog, Bugreev et al. (2007b) hypothesized that it may also bear similarities to Srs2, while $\mathrm{Hu}$ et al. (2007) started with the presumption that the other RecQ helicases may represent potential candidates as the functional equivalent for Srs2 in humans. It turned out that both hypotheses were right, and both BLM (Bugreev et al. 2007b) and RecQL5 (Hu et al. 2007) resemble Srs2 in respect to their ability to disrupt Rad51 filaments, although with some differences that are discussed below.

\section{Similarities and dissimilarities between BLM, RecQL5, and Srs2}

Although the human RecQ helicases share many biochemical activities (Sharma et al. 2006), it appears that the ability to disrupt Rad51 filaments is restricted to BLM (Bugreev et al. 2007b) and RecQL5 (Hu et al. 2007), as WRN (Hu et al. 2007) and RecQL1 (Bugreev et al. 2007b; Hu et al. 2007) were not able to inhibit the D-loop reaction even at concentrations much higher than those used for RecQL5. Although RecQL4 ability to act as a translocase is not yet ruled out, it is important to note that this is the only RecQ inactive as a helicase (Sharma et al. 2006).

Two additional questions come to mind: How similar to the Srs2 activity are the ones now reported for BLM and RecQL5? And are the translocase activities of these two RecQ helicases also implicated in other cellular pathways involving protein-DNA complexes? Like Srs2 (Krejci et al. 2003; Veaute et al. 2003), both BLM's and RecQL5's ATPase activities are strongly stimulated by ssDNA (Bugreev et al. 2007b; Hu et al. 2007). However, while Srs2 can also remove RecA, the Escherichia coli relative of Rad51 (Krejci et al. 2003; Veaute et al. 2003) and hRad51 (Krejci et al. 2003) from DNA, BLM can only remove hRad51, and it is unable to inhibit strand exchange activity of a meiotic hRad51 homolog, hDmc1, or of yeast Rad51 protein (Bugreev et al. 2007b). These results indicate that while Srs2 can recognize a general, common feature of the presynaptic filaments formed by the RecA/Rad51 class of recombinases, the effect of BLM in this reaction is quite specific. Since BLM interacts physically with hRad51 (Wu et al. 2001), the displacement might also involve protein interactions. BLM forms a stable complex with Top3 $\alpha$ (Wu et al. 2000), which is required for $\mathrm{dHJ}$ dissolution (Wu and Hickson 2003), and thus it would be of interest to address whether Top3 affects BLM activity with regard to Rad51 filaments' disruption. Intriguingly, Bugreev et al. (2007b) show that BLM is only able to disrupt inactive hRad51 filaments present in an ADP-bound form. It was previously shown that ATP hydrolysis leads to spontaneous hRad51 filament inactivation (Bugreev and Mazin 2004) and that some auxiliary proteins may help to maintain the filament in an active form (Shim et al. 2004), thus corroborating the idea that hRad51 displacement by BLM occurs if the cells are not fully prepared for the later steps of HR. In this situation, the hRad51 filaments may remain inactive and susceptible to BLM dissociation. In contrast to the limited ability of BLM to disrupt only inactive, ADP-bound Rad51 filaments, $\mathrm{Hu}$ et al. (2007) show that RecQL5 is capable of displacing both Rad51 and Rad51 K133R protein from ssDNA. Rad51 K133R binds ATP and is greatly attenuated for ATP hydrolysis, thus forming a very stable presynaptic filament (Chi et al. 2006). The D-loop disruption activity of RecQL5 is greatly stimulated by the single-stranded binding protein RPA, in a manner reminiscent of Srs2 (Krejci et al. 2003), and Hu et al. (2007) suggest that this enhancement is perhaps due to the ability of RPA to sequester the ssDNA after RecQL5-mediated Rad51 removal, to prevent Rad51 renucleation on DNA (Fig. 1B).

Both Srs2 and RecQ helicases in different organisms have been suggested to play an important role in processing intermediates that arise at damaged or stalled replication forks (Sharma et al. 2006; Sung and Klein 2006). Previous studies have suggested that regulation of Srs 2 is achieved via its recruitment to stalled replication forks through its interaction with sumoylated PCNA (proliferating cell nuclear antigen) (Papouli et al. 2005; Pfander et al. 2005), an important replication and repair factor. Based on genetic evidence, it has been hypothesized that this recruitment favors translesion DNA synthesis or error-free post-replication repair mechanisms that are thought to involve template-switching pathways, and to disfavor HR (Stelter and Ulrich 2003; Papouli et al. 2005; Pfander et al. 2005). BS cells have a prolonged S phase and sensitivity to DNA damaging agents and replication inhibitors, and BLM interacts with several proteins involved in DNA replication, including DNA polymerase $\delta$, RPA, and PCNA (for review, see Sharma et al. 2006). In agreement with a role for BLM in DNA replication, the absence of BLM in Xenopus leads to chromosomal breaks during S phase (Liao et al. 2000; Li et al. 2004), and an accumulation of Rad51-dependent cruciform structures at damaged replication forks in budding yeast sgs1 mutants (Liberi et al. 2005). On the other hand, RECQL5 knockout DT40 cells do not show slow-growth phenotypes and sensitivity to DNA damaging agents (Wang et 
al. 2003), and Recq15 knockout mouse ES cells show only a mild growth defect and a slightly higher level of sensitivity to $\gamma$-rays (Hu et al. 2005). However, recent studies show that RecQL5 also interacts with PCNA and colocalizes with the replication machinery in S-phase nuclei (Kanagaraj et al. 2006). The interactions of BLM and RecQL5 with PCNA thus resemble the one reported for Srs2 (Papouli et al. 2005; Pfander et al. 2005), but at present it is not known whether sumoylation affects this interaction. Nevertheless, both BLM and Sgs1 are sumoylated (Eladad et al. 2005; Branzei et al. 2006). Sumoylation was shown to affect the ability of BLM to localize to PML bodies and suppress SCE accumulation (Eladad et al. 2005), and the ability of Sgs1 to resolve the cruciform structures that arise in a Rad51-dependent manner during replication of damaged templates (Branzei et al. 2006). Interestingly, $\mathrm{Hu}$ et al. (2007) report that in response to camptothecin (CPT)-induced replication stress, Recq15 knockout cells, but not wild-type or Blm knockout ES cells, exhibit a dramatic increase in the frequency of GCRs, and they provide evidence that the GCR phenotype of Recq15 is likely due to failure to regulate HR. Taken together, these findings support the idea that RecQL5 might function preferentially in response to certain types of replication problems (as in CPT-induced intra-S lesions) to prevent HR via Rad51 presynaptic filament disruption (Hu et al. 2007), while BLM might function at least at two different stages in the HR process (Bugreev et al. 2007b) and maybe at replication forks that need to restart, as it has also been suggested for its yeast ortholog, Sgs1 (Liberi et al. 2005).

\section{RecQL5 role in SCE}

The ability of RecQL5 to disrupt presynaptic filaments is likely relevant also for the process leading to SCE formation (Hu et al. 2007). Indeed, previous genetic studies conducted in chicken DT40 cells and mouse ES cells indicated that RecQL5 might function in control of SCEs in a manner that, at least in chicken cells, may be partly redundant to that of BLM (Wang et al. 2003; Hu et al. 2005). Recq5 $5^{-/-}$mouse ES cells display a significantly higher number of SCEs (Hu et al. 2005), while RECQL5 $5^{-/-}$DT40 cells are comparable to wild-type cells for all phenotypes, including SCE levels (Wang et al. 2003). However both chicken and mouse double knockout blm recq15 showed a significant higher number in SCEs (Wang et al. 2003; Hu et al. 2005), pointing to a role of RecQL5 in suppressing crossovers. Recq15 knockout mice are very similar to their wild-type siblings (Hu et al. 2005), except for the striking cancer susceptibility phenotype that Hu et al. (2007) report in this issue. Interestingly, while mutations in both human $B L M$ and mouse Blm result in elevated levels of $\mathrm{LOH}$ (see also above), $\mathrm{Hu}$ et al. (2007) find that Recq15 knockout mouse ES cells have similar frequencies of LOH to wild-type ES cells as measured at two different loci. This finding indicates that Blm and Recql5 contribute through different mechanisms to suppressing crossovers, in agreement with a previous model (Hu et al. 2005). However, the findings in chicken DT40 cells (Wang et al. 2003) indicate there could be differences between organisms with respect to this.

The biochemical functions of RecQL5 also resemble those of BLM in several aspects. The RECQL5 gene encodes three isoforms, but only RecQL5 $\beta$ localizes to the nucleus (Shimamoto et al. 2000). Like BLM, RecQL5 $\beta$ has $3^{\prime}-5^{\prime}$ DNA helicase activity, ssDNA annealing activity, can catalyze branch migration of HJs (Garcia et al. 2004; Kanagaraj et al. 2006), and interacts with Top3 $\alpha$ (Shimamoto et al. 2000). BLM together with Top3 $\alpha$ was shown to catalyze dHJ dissolution on model DNA substrates to lead exclusively to noncrossover products (Wu and Hickson 2003). In this reaction, BLM cannot be substituted by E. coli UvrD helicase, and Top $3 \alpha$ cannot be substituted by human topoisomerase I ( Wu and Hickson 2003). Since genetic and biochemical evidence suggested that RecQL5 might serve as a backup system for BLM (Wang et al. 2003; Garcia et al. 2004; Kanagaraj et al. 2006), one possibility was that also RecQL5 could act in combination with Top3 in dissolving dHJs. However, as Hu et al. (2007) discuss in their study, they could find no evidence for such an activity of RecQL5 even when BLAP75, a component of the BLM/Top3 $\alpha$ complex that enhances BLM activity in $\mathrm{dHJ}$ dissolution (Wu and Hickson 2003; Raynard et al. 2006; Wu et al. 2006), was included in the reaction. The ability of RecQL5 to influence BLM/Top $3 \alpha$ activity in the $\mathrm{dHJ}$ dissolution process remains to be analyzed, but nevertheless these data collectively suggest that RecQL5 acts to regulate SCE formation through a mechanism primarily distinct from dHJ dissolution.

\section{Dissociation of D-loops by Sgs1/BLM: relevance for promoting SDSA and preventing SCE}

Dissociation of the extended D-loop formed in an HR reaction is expected to shunt this intermediate to the SDSA pathway and prevent crossover recombinants. A mechanism through which BLM/Sgs1 acts to prevent crossover is likely through its ability to dissolve dHJs (Fig. 2; Wu and Hickson 2003). Genetic data in yeast and Drosophila are supportive of this model (Adams et al. 2003; Ira et al. 2003), but could also be interpreted with the SDSA model, in which BLM/Sgs1 would prevent crossovers by promoting dissolution of the D-loop intermediate (Fig. 2). Genetic data suggest that also Srs2 is required for efficient DSBR through the SDSA pathway (Aylon et al. 2003; Ira et al. 2003; Robert et al. 2006). However, while biochemical analysis of Srs2 could not yet recapitulate such activity (Veaute et al. 2003), possibly because other cofactors are required, human BLM was shown to dissociate D-loops by itself (van Brabant et al. 2000). Bugreev et al. (2007b) confirm this observation, although they find that again, this activity depends on the conformation of the hRad51 filament; that is, that BLM can efficiently dissociate D-loops following hRad51 inactivation, but is unable to do so when hRad51 is maintained in an active ATP-bound form in the presence of $\mathrm{Ca}^{2+}$. This activity parallels that of hRad54 (Bugreev et 
al. 2007a). Thus, both BLM and hRAD54 could act to promote HR through the SDSA mechanism leading to noncrossover products. This role for BLM in SDSA was also previously proposed based on genetic studies in Drosophila that suggested a model of multiple invasion/dissolution cycles during SDSA, which in the absence of BLM would result in short repair synthesis tracts or flanking deletions (McVey et al. 2004). The findings of Bugreev et al. (2007b) in this issue support this model, and in addition they show that BLM can stimulate polymerase $\eta$-mediated DNA synthesis on a model replication fork, possibly to promote SDSA or in general HR at late stages.

\section{Protein interactions and RecQ-like helicases} in controlling recombination

Although certain biochemical activities and functions of RecQ helicases are partially redundant, the distinct clinical characteristics and cellular phenotypes of RecQ helicase disorders or knockout cells indicate unique roles of these proteins. Increasing evidence suggests that many, if not all, of the RecQ proteins can function both alone or in combination with other proteins, and we suggest that their distinct functions may be achieved through specialized protein interactions or post-translational modifications. This trend is also reflected by biochemical studies. For instance, BLM can dissociate Dloops by itself (van Brabant et al. 2000; Bugreev et al. $2007 b$ ), while dissolution of $\mathrm{dHJ}$ s requires both BLM and Top $3 \alpha$ and is stimulated by BLAP75/Rmil (Wu and Hickson 2003; Raynard et al. 2006; Wu et al. 2006). In support of this view, Bugreev et al. (2007b) show that BLM activities in HR depend on hRad51 protein conformation, which could also be modulated via protein interactions. In addition, BLM opposing roles in stimulating or counteracting HR could also be controlled by the availability of other interacting proteins. Likewise, RecQL5 function in suppressing initial steps of HR during replication ( $\mathrm{Hu}$ et al. 2007) may be regulated by PCNA and RPA.

In addition to the potentially overlapping roles of RecQ helicases, there are additional helicases that, like Srs2, function in the same or parallel pathways and sometimes also show physical interactions or colocalization with one or a subset of RecQ helicases. In respect to the roles of these "RecQ-like" DNA helicases in controlling or promoting $\mathrm{HR}$ and DSBR, we envisage that at least two proteins might deserve special attention for future studies, FBH1 and BACH1. FBH1 shows structural similarity to Srs2, the mutation in $S$. pombe leads to phenotypes and genetic interactions that resemble those of srs2 (Morishita et al. 2005; Osman et al. 2005), and budding yeast srs2 phenotypes can be partly complemented by hFBH1 (Chiolo et al. 2007). In addition, FBH1 mutation in DT40 cells leads to a slight increase in SCE, and in a BLM knockout context, FBH1 disruption in DT40 cells results in a further increase in SCE (Kohzaki et al. 2007), resembling in this aspect RECQL5 (Wang et al. 2003). BACH1, also known as FANCJ and BRIP1, is a
DNA helicase that displays sequence identity with BLM in the DNA helicase domain, and is implicated in maintenance of genome stability, DSBR, and Fanconi anemia (FA) (Kumaraswamy and Shiekhattar 2007; Niedernhofer 2007 and references therein).

Again, the physical and functional links between BLM, HR factors, and FA proteins (Niedernhofer 2007) suggest that these cooperations could be achieved via protein interactions. Also in line with this view, recent findings show that BACH1 is required for timely progress through $S$ phase and its activation is achieved through a dephosphorylation event that occurs as cells enter S phase (Kumaraswamy and Shiekhattar 2007). Based on the available information, we think it likely that the dazzling complexity of the pro- and anti-recombination activities of the RecQ helicases is related to the presence in the cell of other RecQ-like DNA helicases and their engagement in different dynamic protein complexes that could also be influenced or regulated by the cell cycle phase and additional post-translational modifications. The understanding of how these factors affect HR regulation and RecQ biology will be a key challenge for the future.

\section{Acknowledgments}

The work in our laboratories is supported by grants from the Associazione Italiana per la Ricerca sul Cancro, European Community, Telethon-Italy, Italian Ministry of Education, and Association for International Cancer Research to M.F. and D.B. D.B. is supported by the Buzzati-Traverso foundation.

\section{References}

Aboussekhra, A., Chanet, R., Zgaga, Z., Cassier-Chauvat, C., Heude, M., and Fabre, F. 1989. RADH, a gene of Saccharomyces cerevisiae encoding a putative DNA helicase involved in DNA repair. Characteristics of radH mutants and sequence of the gene. Nucleic Acids Res. 17: 7211-7219.

Adams, M.D., McVey, M., and Sekelsky, J.J. 2003. Drosophila BLM in double-strand break repair by synthesis-dependent strand annealing. Science 299: 265-267.

Allers, T. and Lichten, M. 2001. Differential timing and control of noncrossover and crossover recombination during meiosis. Cell 106: 47-57.

Aylon, Y., Liefshitz, B., Bitan-Banin, G., and Kupiec, M. 2003. Molecular dissection of mitotic recombination in the yeast Saccharomyces cerevisiae. Mol. Cell. Biol. 23: 1403-1417.

Bachrati, C.Z., Borts, R.H., and Hickson, I.D. 2006. Mobile Dloops are a preferred substrate for the Bloom's syndrome helicase. Nucleic Acids Res. 34: 2269-2279.

Branzei, D., Sollier, J., Liberi, G., Zhao, X., Maeda, D., Seki, M., Enomoto, T., Ohta, K., and Foiani, M. 2006. Ubc9- and Mms21-mediated sumoylation counteracts recombinogenic events at damaged replication forks. Cell 127: 509-522.

Bugreev, D.V. and Mazin, A.V. 2004. $\mathrm{Ca}^{2+}$ activates human homologous recombination protein Rad51 by modulating its ATPase activity. Proc. Natl. Acad. Sci. 101: 9988-9993.

Bugreev, D.V., Hanaoka, F., and Mazin, A.V. 2007a. Rad54 dissociates homologous recombination intermediates by branch migration. Nat. Struct. Mol. Biol. 14: 746-753.

Bugreev, D.V., Yu, X., Egelman, E.H., and Mazin, A.V. 2007b. 
Novel pro- and anti-recombination activities of the Bloom's syndrome helicase. Genes \& Dev. (this issue). doi: 10.1101/gad. 1609007.

Chi, P., Van Komen, S., Sehorn, M.G., Sigurdsson, S., and Sung, P. 2006. Roles of ATP binding and ATP hydrolysis in human Rad51 recombinase function. DNA Repair (Amst.) 5: 381391.

Chiolo, I., Saponaro, M., Baryshnikova, A., Kim, J.H., Seo, Y.S., and Liberi, G. 2007. The human F-box DNA helicase FBH1 faces $S$. cerevisiae Srs2 and post-replication repair pathway roles. Mol. Cell. Biol. 27: 7439-7450.

Eladad, S., Ye, T.Z., Hu, P., Leversha, M., Beresten, S., Matunis, M.J., and Ellis, N.A. 2005. Intra-nuclear trafficking of the BLM helicase to DNA damage-induced foci is regulated by SUMO modification. Hum. Mol. Genet. 14: 1351-1365.

Gangloff, S., Soustelle, C., and Fabre, F. 2000. Homologous recombination is responsible for cell death in the absence of the Sgs1 and Srs2 helicases. Nat. Genet. 25: 192-194.

Garcia, P.L., Liu, Y., Jiricny, J., West, S.C., and Janscak, P. 2004. Human RECQ5 $\beta$, a protein with DNA helicase and strandannealing activities in a single polypeptide. $E M B O ~ J .23$ : 2882-2891.

German, J. 1993. Bloom syndrome: A Mendelian prototype of somatic mutational disease. Medicine (Baltimore) 72: 393 406.

Goss, K.H., Risinger, M.A., Kordich, J.J., Sanz, M.M., Straughen, J.E., Slovek, L.E., Capobianco, A.J., German, J., Boivin, G.P., and Groden, J. 2002. Enhanced tumor formation in mice heterozygous for Blm mutation. Science 297: 2051-2053.

Hanada, K. and Hickson, I.D. 2007. Molecular genetics of RecQ helicase disorders. Cell. Mol. Life Sci. 64: 2306-2322.

Hu, Y., Lu, X., Barnes, E., Yan, M., Lou, H., and Luo, G. 2005. Recql5 and Blm RecQ DNA helicases have nonredundant roles in suppressing crossovers. Mol. Cell. Biol. 25: 34313442.

Hu, Y., Raynard, S., Sehorn, M.G., Lu, X., Bussen, W., Zheng, L., Stark, J.M., Barnes, E.L., Chi, P., Janscak, P., et al. 2007. RECQL5/Recq15 helicase regulates homologous recombination and suppresses tumor formation via disruption of Rad51 presynaptic filaments. Genes \& Dev. (this issue). doi: 10.1101/ gad.1609107.

Hunter, N. and Kleckner, N. 2001. The single-end invasion: An asymmetric intermediate at the double-strand break to double-Holliday junction transition of meiotic recombination. Cell 106: 59-70.

Ira, G., Malkova, A., Liberi, G., Foiani, M., and Haber, J.E. 2003. Srs2 and Sgs1-Top3 suppress crossovers during doublestrand break repair in yeast. Cell 115: 401-411.

Johnson-Schlitz, D. and Engels, W.R. 2006. Template disruptions and failure of double Holliday junction dissolution during double-strand break repair in Drosophila BLM mutants. Proc. Natl. Acad. Sci. 103: 16840-16845.

Kanagaraj, R., Saydam, N., Garcia, P.L., Zheng, L., and Janscak, P. 2006. Human RECQ5 $\beta$ helicase promotes strand exchange on synthetic DNA structures resembling a stalled replication fork. Nucleic Acids Res. 34: 5217-5231.

Kohzaki, M., Hatanaka, A., Sonoda, E., Yamazoe, M., Kikuchi, K., Vu Trung, N., Szuts, D., Sale, J.E., Shinagawa, H., Watanabe, M., et al. 2007. Cooperative roles of vertebrate Fbh1 and Blm DNA helicases in avoidance of crossovers during recombination initiated by replication fork collapse. Mol. Cell. Biol. 27: 2812-2820.

Krejci, L., Van Komen, S., Li, Y., Villemain, J., Reddy, M.S., Klein, H., Ellenberger, T., and Sung, P. 2003. DNA helicase Srs2 disrupts the Rad51 presynaptic filament. Nature 423: 305-309.
Kumaraswamy, E. and Shiekhattar, R. 2007. Activation of BRCA1/BRCA2-associated helicase BACH1 is required for timely progression through S phase. Mol. Cell. Biol. 27: 6733-6741.

Li, W., Kim, S.M., Lee, J., and Dunphy, W.G. 2004. Absence of BLM leads to accumulation of chromosomal DNA breaks during both unperturbed and disrupted S phases. J. Cell Biol. 165: 801-812.

Liao, S., Graham, J., and Yan, H. 2000. The function of Xenopus Bloom's syndrome protein homolog (xBLM) in DNA replication. Genes \& Dev. 14: 2570-2575.

Liberi, G., Maffioletti, G., Lucca, C., Chiolo, I., Baryshnikova, A., Cotta-Ramusino, C., Lopes, M., Pellicioli, A., Haber, J.E., and Foiani, M. 2005. Rad51-dependent DNA structures accumulate at damaged replication forks in sgs 1 mutants defective in the yeast ortholog of BLM RecQ helicase. Genes \& Dev. 19: 339-350.

Luo, G., Santoro, I.M., McDaniel, L.D., Nishijima, I., Mills, M., Youssoufian, H., Vogel, H., Schultz, R.A., and Bradley, A. 2000. Cancer predisposition caused by elevated mitotic recombination in Bloom mice. Nat. Genet. 26: 424-429.

Mankouri, H.W., Craig, T.J., and Morgan, A. 2002. SGS1 is a multicopy suppressor of srs2: Functional overlap between DNA helicases. Nucleic Acids Res. 30: 1103-1113.

McVey, M., Larocque, J.R., Adams, M.D., and Sekelsky, J.J. 2004. Formation of deletions during double-strand break repair in Drosophila DmBlm mutants occurs after strand invasion. Proc. Natl. Acad. Sci. 101: 15694-15699.

Morishita, T., Furukawa, F., Sakaguchi, C., Toda, T., Carr, A.M., Iwasaki, H., and Shinagawa, H. 2005. Role of the Schizosaccharomyces pombe F-Box DNA helicase in processing recombination intermediates. Mol. Cell. Biol. 25: 8074-8083.

Niedernhofer, L.J. 2007. The Fanconi anemia signalosome anchor. Mol. Cell 25: 487-490.

Osman, F., Dixon, J., Barr, A.R., and Whitby, M.C. 2005. The F-Box DNA helicase Fbh1 prevents Rhp51-dependent recombination without mediator proteins. Mol. Cell. Biol. 25: 8084-8096.

Papouli, E., Chen, S., Davies, A.A., Huttner, D., Krejci, L., Sung, P., and Ulrich, H.D. 2005. Crosstalk between SUMO and ubiquitin on PCNA is mediated by recruitment of the helicase Srs2p. Mol. Cell 19: 123-133.

Paques, F. and Haber, J.E. 1999. Multiple pathways of recombination induced by double-strand breaks in Saccharomyces cerevisiae. Microbiol. Mol. Biol. Rev. 63: 349-404.

Pfander, B., Moldovan, G.L., Sacher, M., Hoege, C., and Jentsch, S. 2005. SUMO-modified PCNA recruits Srs2 to prevent recombination during S phase. Nature 436: 428-433.

Ray, J.H. and German, J. 1984. Bloom's syndrome and EM9 cells in BrdU-containing medium exhibit similarly elevated frequencies of sister chromatid exchange but dissimilar amounts of cellular proliferation and chromosome disruption. Chromosoma 90: 383-388.

Raynard, S., Bussen, W., and Sung, P. 2006. A double Holliday junction dissolvasome comprising BLM, topoisomerase III $\alpha$, and BLAP75. J. Biol. Chem. 281: 13861-13864.

Robert, T., Dervins, D., Fabre, F., and Gangloff, S. 2006. Mrc1 and Srs2 are major actors in the regulation of spontaneous crossover. EMBO T. 25: 2837-2846.

Rong, L. and Klein, H.L. 1993. Purification and characterization of the SRS2 DNA helicase of the yeast Saccharomyces cerevisiae. J. Biol. Chem. 268: 1252-1259.

Schiestl, R.H., Prakash, S., and Prakash, L. 1990. The SRS2 suppressor of rad6 mutations of Saccharomyces cerevisiae acts by channeling DNA lesions into the RAD52 DNA repair 
pathway. Genetics 124: 817-831.

Sharma, S., Doherty, K.M., and Brosh Jr., R.M. 2006. Mechanisms of RecQ helicases in pathways of DNA metabolism and maintenance of genomic stability. Biochem. J. 398: 319337.

Shim, K.S., Schmutte, C., Tombline, G., Heinen, C.D., and Fishel, R. 2004. hXRCC2 enhances ADP/ATP processing and strand exchange by hRAD51. J. Biol. Chem. 279: 3038530394.

Shimamoto, A., Nishikawa, K., Kitao, S., and Furuichi, Y. 2000. Human RecQ5 $\beta$, a large isomer of RecQ5 DNA helicase, localizes in the nucleoplasm and interacts with topoisomerases $3 \alpha$ and $3 \beta$. Nucleic Acids Res. 28: 1647-1655.

Sonoda, E., Sasaki, M.S., Morrison, C., Yamaguchi-Iwai, Y., Takata, M., and Takeda, S. 1999. Sister chromatid exchanges are mediated by homologous recombination in vertebrate cells. Mol. Cell. Biol. 19: 5166-5169.

Stelter, P. and Ulrich, H.D. 2003. Control of spontaneous and damage-induced mutagenesis by SUMO and ubiquitin conjugation. Nature 425: 188-191.

Sung, P. and Klein, H. 2006. Mechanism of homologous recombination: Mediators and helicases take on regulatory functions. Nat. Rev. Mol. Cell Biol. 7: 739-750.

Sung, P. and Robberson, D.L. 1995. DNA strand exchange mediated by a RAD51-ssDNA nucleoprotein filament with polarity opposite to that of RecA. Cell 82: 453-461.

Thompson, L.H. and Schild, D. 2002. Recombinational DNA repair and human disease. Mutat. Res. 509: 49-78.

van Brabant, A.J., Ye, T., Sanz, M., German, I.J., Ellis, N.A., and Holloman, W.K. 2000. Binding and melting of D-loops by the Bloom syndrome helicase. Biochemistry 39: 14617-14625.

Veaute, X., Jeusset, J., Soustelle, C., Kowalczykowski, S.C., Le Cam, E., and Fabre, F. 2003. The Srs2 helicase prevents recombination by disrupting Rad51 nucleoprotein filaments. Nature 423: 309-312.

Wang, W., Seki, M., Narita, Y., Sonoda, E., Takeda, S., Yamada, K., Masuko, T., Katada, T., and Enomoto, T. 2000. Possible association of BLM in decreasing DNA double strand breaks during DNA replication. EMBO J. 19: 3428-3435.

Wang, W., Seki, M., Narita, Y., Nakagawa, T., Yoshimura, A., Otsuki, M., Kawabe, Y., Tada, S., Yagi, H., Ishii, Y., et al. 2003. Functional relation among RecQ family helicases RecQL1, RecQL5, and BLM in cell growth and sister chromatid exchange formation. Mol. Cell. Biol. 23: 3527-3535.

Wu, L. and Hickson, I.D. 2003. The Bloom's syndrome helicase suppresses crossing over during homologous recombination. Nature 426: 870-874.

Wu, L., Davies, S.L., North, P.S., Goulaouic, H., Riou, J.F., Turley, H., Gatter, K.C., and Hickson, I.D. 2000. The Bloom's syndrome gene product interacts with topoisomerase III. $J$. Biol. Chem. 275: 9636-9644.

Wu, L., Davies, S.L., Levitt, N.C., and Hickson, I.D. 2001. Potential role for the BLM helicase in recombinational repair via a conserved interaction with RAD51. J. Biol. Chem. 276: 19375-19381.

Wu, L., Bachrati, C.Z., Ou, J., Xu, C., Yin, J., Chang, M., Wang, W., Li, L., Brown, G.W., and Hickson, I.D. 2006. BLAP75/ RMI1 promotes the BLM-dependent dissolution of homologous recombination intermediates. Proc. Natl. Acad. Sci. 103: 4068-4073. 


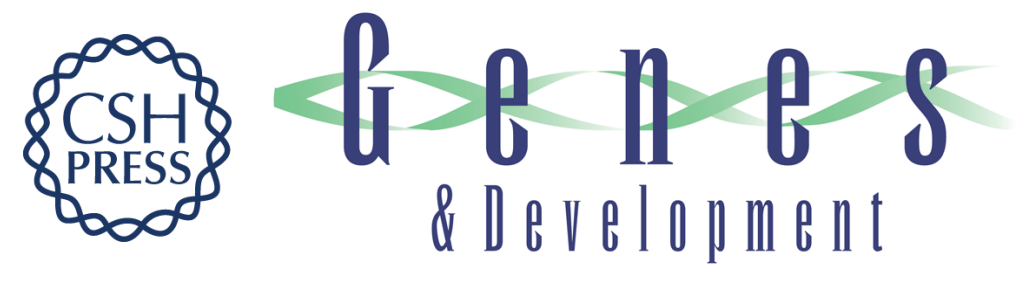

\section{RecQ helicases queuing with Srs2 to disrupt Rad51 filaments and suppress recombination}

Dana Branzei and Marco Foiani

Genes Dev. 2007, 21:

Access the most recent version at doi:10.1101/gad.1624707

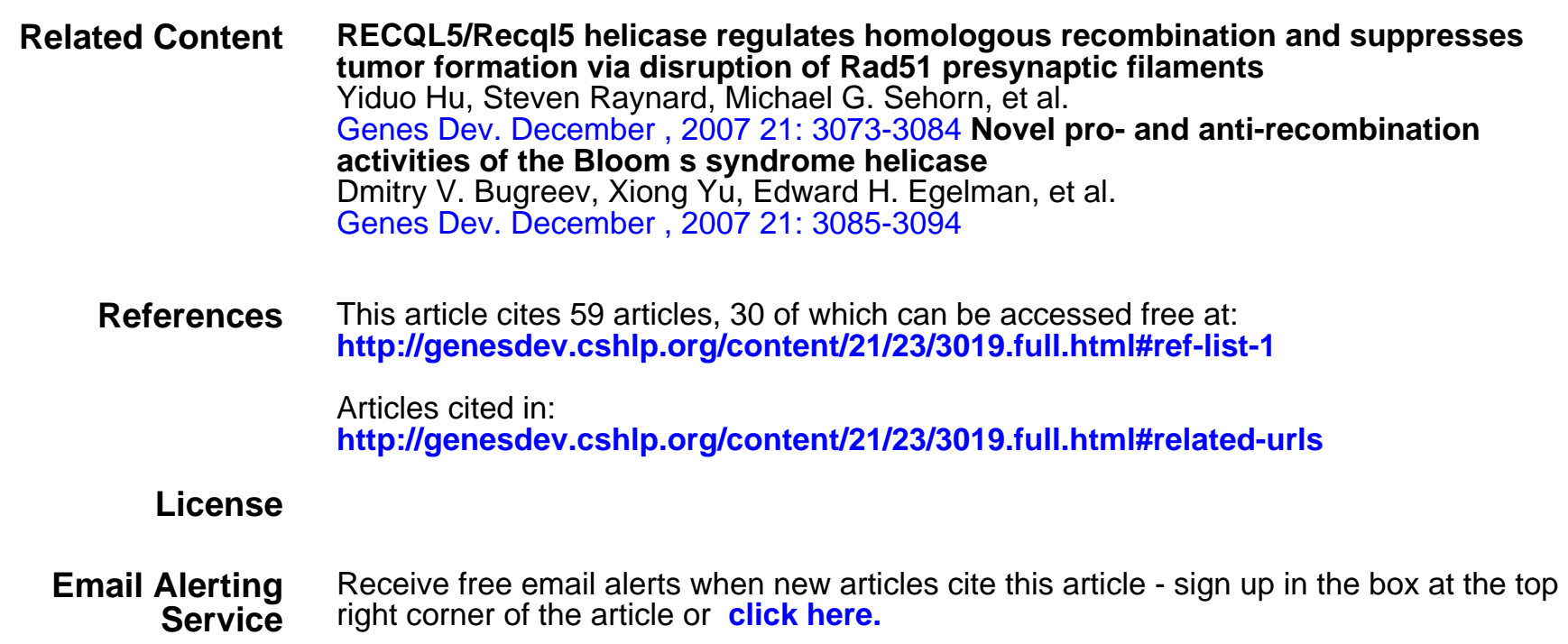

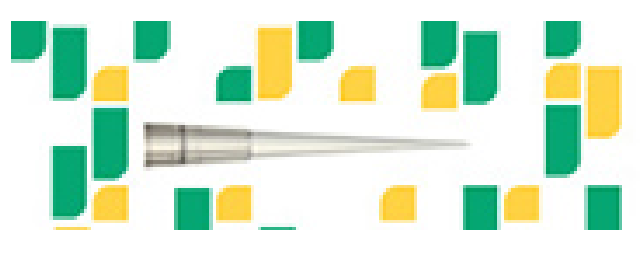

Focused on your science. 